\title{
Impact of BRCA Mutation on the Survival and Risk of Contralateral Breast Cancer in Asian Breast Cancer Patients
}

Po-Han Lin ( $\sim$ pohanlin01@gmail.com )

National Taiwan University Hospital https://orcid.org/0000-0001-6066-398X

Shin-Cheh Chen

Chang Gung Memorial Hospital

Ling-Ming Tseng

Taipei Veterans General Hospital

King-Jen Chang

National Taiwan University Hospital

Ai-Chu Huang

National Taiwan University Hospital

Kuo-Chih Cheng

National Taiwan University Hospital

Karen Yang

National Taiwan University Hospital

Hui-Chen Wu

Columbia University Mailman School of Public Health

Tsu-Yi Chao

Taipei Medical University

Yuan-Ching Chang

Mackay Memorial Hospital

Peng-Chan Lin

National Cheng Kung University

Wen-Hung Kuo

National Taiwan University Hospital

Wen-Lin Kuo

Chang Gung Memorial Hospital

Ching-Hung Lin

National Taiwan University Hospital

Huo-Mu Chen

National Taiwan University Hospital

Dah-Cherng Yeh 
Cheng Ching Hospital Chung Kang Branch: Cheng Ching Hospital

\section{Liang-Chih Liu}

China Medical University Hospital

\section{Chun-Yu Liu}

China Medical University Hospital

\section{Ming-Yang Wang}

National Taiwan University Hospital

Chiao Lo

National Taiwan University Hospital

Yen-Shen Lu

National Taiwan University Hospital

\section{Chiun-Sheng Huang}

National Taiwan University Hospital

\section{Research Article}

Keywords: Asian women, BRCA1, BRCA2, contralateral breast cancer, survival

Posted Date: March 10th, 2021

DOI: https://doi.org/10.21203/rs.3.rs-295032/v1

License: (c) (i) This work is licensed under a Creative Commons Attribution 4.0 International License. Read Full License

Version of Record: A version of this preprint was published at Breast Cancer Research and Treatment on February 3rd, 2022. See the published version at https://doi.org/10.1007/s10549-021-06446-7. 


\section{Abstract}

\section{Purpose}

Breast cancer is increasing around the globe, including Asia. We aimed to examine the survival and risk of contralateral breast cancer $(\mathrm{CBC})$ in Asian breast cancer patients with $B R C A$ mutations.

\section{Methods}

A total of 128 breast cancer patients with germline BRCA mutations and 4,754 control breast cancer patients were enrolled. Data on clinical pathologic characteristics, survival and CBC were collected from the medical record. The rates of survival and CBC were estimated by Kaplan-Meier method.

\section{Results}

The mean age of onset in $B R C A$ mutation carriers was significantly younger than control patients ( $B R C A$ vs. Non-BRCA: 43.9 vs. 53.2 years-old). $B R C A$ mutation carriers had a higher proportion of triple-negative breast cancer (TNBC) $(52 \%)$ than control patients $(12 \%, p<0.001)$. The risk of CBC was significantly higher in $B R C A$ mutation patients than in control cases (hazard ratio (HR) $=3.95,95 \% \mathrm{Cl} 2.71-5.75$ ); when stratified by genotype, the HRs $(95 \% \mathrm{Cl})$ were 4.84 (3.00-7.82) for BRCA1 and 3.13 (1.78-5.49) for BRCA2 carriers, respectively. Moreover, BRCA1 mutation patients with triple-negative breast cancer (TNBC) as their first breast cancer had the highest risk of CBC (HR $=5.55,95 \% \mathrm{Cl} 3.29-9.34)$. However, we did not observe any differences in relapse-free survival and overall survival between mutation carriers and control patients.

\section{Conclusion}

Our study suggest that $B R C A$ patients had a significantly higher risk of developing $\mathrm{CBC}$, particularly for $B R C A 1$ mutation carriers with TNBC as the first breast cancer.

\section{Introduction}

Breast cancer, the most common type of malignancy, is the leading cause of cancer-related death of women in Taiwan and worldwide [1]. One phenomenon of Asian breast cancer patients is that they have a relatively younger onset age than Western patients [2,3], which suggests that a proportion of Asian breast cancer patients is being affected by the genetic mutation of inherited genes such as BRCA1 and BRCA2 $[2,3]$.

Women with germline BRCA mutations have extremely high lifetime risks of developing breast and ovarian cancers [4-7]. Previous studies have shown that the cumulative incidence of breast cancer for $B R C A 1$ and BRCA2-mutation carriers by 80 years old was about $72 \%$ [confidence interval $(\mathrm{Cl}) 65 \%-79 \%$ ] and $69 \%(61 \%-77 \%)$, respectively [7]. More specifically, reports from Asian BRCA studies revealed that $B R C A 1$ and BRCA2 mutation carriers have a $49-54 \%$ and $35-48 \%$ chance of developing breast cancer, 
respectively [6, 7]. The risk of recurrent or contralateral breast cancer (CBC) also significantly increases in $B R C A$ mutation carriers compared to non-BRCA carrier patients $[4,8-10]$. The prognostic impact of germline $B R C A$ mutations in breast cancer patients is controversial. A prospective study revealed similar prognoses in patients with and without BRCA mutations [11]. In contrast, another large cohort and metaanalysis studies suggested that $B R C A$ mutations were associated with worse overall survival $[12,13]$.

However, the majority of studies to date focused on the clinical manifestation of $B R C A$ mutations are from European, North American, African or African-American populations. Although the number of reports from Asian populations have been increasing, most are focused on the prevalence and epidemiology of $B R C A[2,3,14-18]$. The clinical characteristics, pathologic features, prognosis, and risk of $\mathrm{CBC}$ remain uncertain in Asian patients with $B R C A$ mutated breast cancer. To estimate the survival and risk of $C B C$, we used information from a total of 4,882 Taiwanese (ethnic Han Chinese) breast cancer patients. Among them, one-hundred and twenty-eight cases were carriers of germline BRCA1 or BRCA2 mutations.

\section{Patients And Method}

\section{Patient}

We collected 128 breast cancer patients who carried germline BRCA1 or BRCA2 mutations during the period 1995-2019 from previous academic studies and hospital-based gene diagnoses [2,3]. The criteria used to select patients for genetic studies or to recommend them for genetic diagnosis was based mainly on NCCN guidelines, which consider factors such as young-onset age, significant familial history and whether age at TNBC diagnosis is less than 60 years old [19]. Ninety patients were diagnosed in National Taiwan University Hospital (NTUH); the remaining were diagnosed at other Taiwanese hospitals.

The control group came from NTUH breast cancer patients. In NTUH's cancer registry database, we found a total of 6,948 patients during the period 1995-2019. First, we excluded 1,470 patients who had incomprehensive data, which means that some fields, such as tumor size or lymph node status, may have been lacking. Second, we excluded patients with BRCA mutations and patients who had family history of breast cancer and/or ovarian cancer in their first/second relatives. This strategy eliminates $B R C A$ mutations in control patients as much as possible. A final total of 4,754 breast cancer patients were enrolled as the control patient group.

All clinical, pathologic data and survival information were recorded from medical charts. The presence of estrogen receptors (ER), progesterone receptors (PR), and Her2 were determined by immunohistochemical staining. The presence of ER or PR was considered negative when less than $1 \%$ of the tumor cells showed positive staining. Positive expression of hormone receptors was classified as luminal type breast cancer, which can be further divided into luminal $A$ and $B$ classifications. We classified breast tumors with PR expression less than $20 \%$, Ki67 more than $20 \%$ and/or histologic grade III as Luminal B type[20, 21]. For Her2 staining, a score of 0 or $1+$ was considered negative; specimens with a score of $2+$ were confirmed 
by fluorescence in situ hybridization analysis. The tumor histological grade was defined using the Nottingham combined histological grading system.

\section{Statistics}

We used the chi-squared test and Fisher's exact test to calculate the significance of variances between each group. We calculated and compared the survival by different groups using Kaplan-Meier analysis. Followup (in years) was considered as the time interval between the date of breast cancer diagnosis and the date of these endpoints: date of CBC diagnosis, date of death, latest date of follow up, or end of followup (December 31, 2019), whichever came first. Cox proportional hazards regression analysis was used to estimate the hazards ratios of survival and second breast cancer and corresponding $95 \%$ confidence interval $(\mathrm{Cl})$ for various factors, including tumor size, lymph node status, molecular types and genotype of BRCA. Statistical analyses were performed using SPSS software (version 17.0) and all $p$ values are two-sided, and $p$-values less than 0.05 are considered statistically significant.

\section{Results}

\section{Clinical characteristics}

Clinical and pathologic characteristics are shown in Table 1. Sixty-eight patients had BRCA1 mutations while 60 patients had $B R C A 2$ mutations. Patients with $B R C A$ mutations had a significantly younger age of onset than that of control patients ( $B R C A$ vs. control: 43.9 vs. 52.5 years-old, $\mathrm{p}<0.001$ ). Additionally, molecular types between $B R C A$ and non- $B R C A$ patients were significantly different $(\mathrm{p}<0.001)$; a higher proportion of $B R C A$ mutation patients than non-BRCA patients had TNBC (BRCA vs. control: $52.3 \%$ vs. $12.0 \%)$, followed by the ER(+) Her2(-) patients (41.4\% vs. $61.3 \%)$. A small proportion of BRCA patients had ER(+) Her2(+) breast cancer (5.5\% vs. $11.1 \%$ ), and none of them had pure Her2 type cancer (0 vs. 10.4\%). When stratified by genotype, $76 \%(n=56)$ of BRCA1 patients had TNBC whereas $66.7 \%(n=40)$ of $B R C A 2$ patients had ER(+) Her2(-) breast cancer. 
Table 1

Patient and tumor characteristics according to germline $B R C A$ status.

\begin{tabular}{|c|c|c|c|c|c|}
\hline Characteristics & $\begin{array}{l}B R C A \\
\text { mutated }(n= \\
128)\end{array}$ & $\begin{array}{l}\text { BRCA1 } \\
\text { mutated } \\
(n=68)\end{array}$ & $\begin{array}{l}\text { BRCA2 } \\
\text { mutated (n } \\
=60)\end{array}$ & $\begin{array}{l}\text { BRCA non- } \\
\text { mutated }(\mathrm{n}= \\
4754)\end{array}$ & $\begin{array}{l}B R C A \text { vs. } \\
\text { non-BRCA } \\
p \text { value }\end{array}$ \\
\hline Age (years, mean $\pm S D$ ) & $43.9 \pm 10.1$ & $\begin{array}{l}42.4 \pm \\
10.2\end{array}$ & $45.6 \pm 9.8$ & $53.2 \pm 11.6$ & $<0.001$ \\
\hline Molecular type & & & & & $<0.001$ \\
\hline ER(+)Her2(-) $)^{\text {LuminalA }}$ & 37 & 8 & 29 & 1813 & \\
\hline $\mathrm{ER}(+)$ Her2(-) $)^{\text {LuminalB }}$ & 16 & 5 & 11 & 793 & \\
\hline ER(+)Her2(+) & 7 & 3 & 4 & 470 & \\
\hline Her2(+) & 0 & 0 & 0 & 456 & \\
\hline TNBC & 67 & 52 & 15 & 514 & \\
\hline unknown & 1 & 0 & 1 & 704 & \\
\hline \multicolumn{6}{|l|}{$\begin{array}{l}\text { Disease status of } \\
\text { diagnosis }\end{array}$} \\
\hline (de novo) stage IV & 4 & 3 & 1 & 250 & 0.185 \\
\hline \multicolumn{6}{|l|}{ Stage 0-III } \\
\hline T classification & & & & & 0.713 \\
\hline T0 & 2 & 1 & 1 & 154 & \\
\hline T1 & 58 & 33 & 25 & 1919 & \\
\hline T2 & 51 & 27 & 24 & 2029 & \\
\hline T3-4 & 10 & 3 & 7 & 402 & \\
\hline unknown & 4 & 2 & 2 & 0 & \\
\hline N classification (post) & & & & & 0.814 \\
\hline NO & 75 & 43 & 32 & 2767 & \\
\hline N1 & 25 & 13 & 12 & 1078 & \\
\hline N2 & 12 & 4 & 8 & 408 & \\
\hline N3 & 7 & 3 & 4 & 210 & \\
\hline unknown & 6 & 3 & 3 & 0 & \\
\hline
\end{tabular}




\begin{tabular}{|llllll|}
\hline Characteristics & $\begin{array}{l}\text { BRCA } \\
\text { mutated }(\mathrm{n}=\end{array}$ & $\begin{array}{l}\text { BRCA1 } \\
\text { mutated } \\
(\mathrm{n}=68)\end{array}$ & $\begin{array}{l}\text { BRCA2 } \\
\text { mutated }(\mathrm{n}) \\
=60)\end{array}$ & $\begin{array}{l}\text { BRCA non- } \\
\text { mutated }(\mathrm{n}= \\
\mathbf{4 7 5 4})\end{array}$ & $\begin{array}{l}\text { BRCA vs. } \\
\text { non-BRCA } \\
p \text { value }\end{array}$ \\
\hline $\begin{array}{l}\text { Second malignancies } \\
\text { (not Breast cancer) }\end{array}$ & & & & 238 & $<0.001$ \\
\hline Positive & 20 & 10 & 10 & 8 & $<0.001$ \\
\hline Ovarian cancer & 17 & 9 & 8 & 230 & $<0.001$ \\
\hline Other cancers & 3 & 2 & 1 & $<0.001$ \\
\hline
\end{tabular}

There were no difference in the distributions of tumor size and $L N$ status between $B R C A$ and non-BRCA mutation patients. $B R C A$ mutation patients had a significantly higher risk of developing second nonbreast cancer $(p<0.001)$. The main second malignancy was ovarian cancer, whose incidence was similar between $B R C A 1$ and $B R C A 2$ patients. For the 17 patients with ovarian cancer, twelve (70.6\%) of them developed ovarian cancer after breast cancer and 5 cases had ovarian cancer before breast cancer. The types of the second malignancy for the control cases were significantly different from that of $B R C A$ patients; the most common cancer of the control patients was lung cancer $(p<0.001)$.

\section{Relapse-free And Overall Survival}

The median follow time was 4.67 (range 0.1 to 22.9) years. Kaplan-Meier analysis revealed that relapsefree survival (RFS) for patients with stage 0 to III breast cancer was similar between BRCA and control patients (Fig. 1A, p = 0.967). The RFS for BRCA patients was $85.2 \%$ ( $95 \%$ confidence interval $(\mathrm{Cl}) 92.2 \%-$ $78.1 \%)$ at five years and $78.5 \%(95 \% \mathrm{Cl} 87.5 \%-69.5 \%)$ at 10 years. On the other hand, the RFS for control patients was $86.2 \%(95 \% \mathrm{Cl} 87.4 \%-85.0 \%)$ and $79.0 \%(95 \% \mathrm{Cl} 80.8 \%-77.2 \%)$ at 5 and 10 years, respectively. When stratified by BRCA1 and BRCA2 genotyping, the 10-year RFS for BRCA1 patients was $82.6 \%$ (95\% $\mathrm{Cl} 71.2 \%$ - 93.9\%) whereas the RFS for BRCA2 patients was $74.4 \%$ (95 $\mathrm{Cl} 60.1 \%-88.3 \%$ ). There was no significant difference in RFS between BRCA1, BRCA2 and control patients (Fig. 1B).

The prognostic factors for RFS in both $B R C A$ and control patients were the tumor size and LN classification (Figs. 1C-1F). Large tumor sizes and advanced LN classification resulted in poor RFS. When stratified by molecular types, the control patients with ER(+) Her2(-) LuminalA breast cancer had the best RFS out of all other types of breast cancer. Patients with ER(-)Her2(+) breast cancer had the worst RFS (Fig. 1G). For BRCA mutation carriers, patients with TNBC had a better RFS trend than those with other types of breast cancer $($ Fig. $1 \mathrm{H})$, probably because most TNBC patients had stage I breast cancer.

Among the entire cohort, tumor size, LN classification and molecular types were independent risk factors for RFS (Table 2). Thus, we analyzed the impact of $B R C A$ after adjusting for these major prognostic factors in a multivariable model; the results showed no difference in RFS between $B R C A$ and control 
patients ( $\mathrm{HR}=1.071,95 \% \mathrm{Cl} 0.689-1.664$, Table 2). In contrast, large tumor size, advanced LN status, ER(+) Her2(-) ${ }^{\text {LuminalB }}$, ER(-) Her2(+) and TNBC remained the independent prognostic factors for RFS. Similarly, there was no difference in the overall survival (OS) between BRCA and control patients (HR= $0.721,95 \% \mathrm{Cl}=0.396-1.314)$. The tumor size, $\mathrm{LN}$ classification and molecular types were the independent risk factors of OS. Compared to patients with $\mathrm{ER}(+)$ Her2(-) $)^{\mathrm{LuminalA}}$, patients with $\mathrm{ER}(+)$ Her2(-) $)^{\text {LuminalB }}$, ER(-)Her2(+) or TNBC had a significantly inferior OS (Table 3). 
Table 2

Univariate and multivariate analysis of relapse-free survival

\begin{tabular}{|c|c|c|c|c|c|c|c|c|}
\hline Clinical & Univariate & & & & Multivaria & & & \\
\hline Characteristics & $\mathrm{HR}$ & lower & upper & $P$ & $\mathrm{HR}$ & lower & upper & $P$ \\
\hline \multicolumn{9}{|l|}{ Tumor size } \\
\hline T0-1 & reference & & & & reference & & & \\
\hline T2 & 2.653 & 2.180 & 3.228 & $\begin{array}{l}<.001 \\
0.00\end{array}$ & 1.864 & 1.516 & 2.291 & $\begin{array}{l}<.001 \\
0.001\end{array}$ \\
\hline T3-4 & 5.594 & 4.370 & 7.160 & $\begin{array}{l}<.001 \\
0.00\end{array}$ & 3.387 & 2.590 & 4.428 & $\begin{array}{l}<.001 \\
0.001\end{array}$ \\
\hline \multicolumn{9}{|l|}{ LN } \\
\hline NO & reference & & & & reference & & & \\
\hline N1 & 2.124 & 1.748 & 2.582 & $\begin{array}{l}<.001 \\
0.00\end{array}$ & 2.131 & 1.732 & 2.622 & $\begin{array}{l}< \\
0.001\end{array}$ \\
\hline N2 & 4.234 & 3.426 & 5.231 & $\begin{array}{l}< \\
0.001\end{array}$ & 3.732 & 2.966 & 4.695 & $\begin{array}{l}<.001 \\
0.001\end{array}$ \\
\hline N3 & 6.976 & 5.474 & 8.891 & $<.001$ & 4.914 & 3.747 & 6.445 & $\begin{array}{l}< \\
0.001\end{array}$ \\
\hline \multicolumn{9}{|l|}{ Molecular type } \\
\hline ER(+)Her2(-) LuminalA & reference & & & & reference & & & \\
\hline $\mathrm{ER}(+) \mathrm{Her} 2(-)^{\text {LuminalB }}$ & 1.399 & 1.138 & 1.720 & 0.001 & 1.302 & 1.053 & 1.610 & 0.015 \\
\hline ERHer2(+) & 1.380 & 1.060 & 1.798 & 0.017 & 1.017 & .770 & 1.345 & 0.903 \\
\hline Her2(+) & 1.885 & 1.478 & 2.405 & $<.001$ & 1.573 & 1.218 & 2.032 & 0.001 \\
\hline TNBC & 1.276 & .990 & 1.643 & 0.060 & 1.304 & 1.001 & 1.699 & 0.049 \\
\hline \multicolumn{9}{|l|}{ Genotyping } \\
\hline non- $B R C A$ & reference & & & & reference & & & \\
\hline$B R C A$ & 0.991 & 0.647 & 1.517 & 0.967 & 1.071 & 0.689 & 1.664 & 0.761 \\
\hline
\end{tabular}


Table 3

Multivariate analysis of overall survival

\begin{tabular}{|c|c|c|c|c|}
\hline Clinical & Multivariate & & & \\
\hline Characteristics & $\mathrm{HR}$ & lower & upper & $P$ \\
\hline \multicolumn{5}{|l|}{ Tumor size } \\
\hline T0-1 & reference & & & \\
\hline $\mathrm{T} 2$ & 1.686 & 1.318 & 2.157 & $<0.001$ \\
\hline T3-4 & 2.503 & 1.753 & 3.576 & $<0.001$ \\
\hline \multicolumn{5}{|l|}{ LN } \\
\hline No & reference & & & \\
\hline N1 & 1.859 & 1.440 & 2.400 & $<0.001$ \\
\hline N2 & 3.170 & 2.390 & 4.204 & $<0.001$ \\
\hline N3 & 3.996 & 2.818 & 5.665 & $<0.001$ \\
\hline \multicolumn{5}{|l|}{ Molecular type } \\
\hline ER(+)Her2(-) LuminalA & reference & & & \\
\hline ER(+)Her2(-) LuminalB & 1.729 & 1.338 & 2.235 & $<0.001$ \\
\hline $\mathrm{ER}(+) \mathrm{Her} 2(+)$ & 1.107 & 0.759 & 1.615 & 0.598 \\
\hline ER(-)Her2(+) & 1.537 & 1.084 & 2.180 & 0.016 \\
\hline TNBC & 1.681 & 1.203 & 2.348 & 0.002 \\
\hline \multicolumn{5}{|l|}{ Genotyping } \\
\hline non- $B R C A$ & reference & & & \\
\hline$B R C A$ & 0.721 & 0.396 & 1.314 & 0.286 \\
\hline
\end{tabular}

\section{Risk Of Contralateral Breast Cancer}

The risk of contralateral breast cancer (CBC) was significantly higher in BRCA mutation patients than in control cases $(\mathrm{HR}=3.950,95 \% \mathrm{Cl} 2.712-5.752, \mathrm{p}<0.001$.. The $\mathrm{CBC}$ risk was $26.0 \%(95 \% \mathrm{Cl} 16.0 \%-$ $36.0 \%)$ at 10 years and $44.0 \%(95 \% \mathrm{Cl} 27.1 \%-60.8 \%)$ at 15 years in BRCA mutation patients, compared to $6.6 \%(95 \% \mathrm{Cl} 5.6 \%-7.6 \%)$ at 10 years and $10.4 \%(95 \% \mathrm{Cl} 8.2 \%-12.6 \%)$ at 15 years in control patients (Fig. 2A). When stratified y BRCA1 and BRCA2 genotyping, both patient groups had a significantly increased risk of $\mathrm{CBC}$ in comparison to control patients $(B R C A 1$ : $\mathrm{HR}=4.844,95 \% \mathrm{Cl} 3.001-7.820, \mathrm{p}<$ 
$0.001 ; B R C A 2: \mathrm{HR}=3.130,95 \% \mathrm{Cl} 1.782-5.498, \mathrm{p}<0.001)$. We then classified patients by molecular types; both $\mathrm{ER}(+)$ and TNBC $B R C A$ mutation patients had a significantly higher risk of getting $\mathrm{CBC}$ than control patients with $\mathrm{ER}(+)$ breast cancer and TNBC, respectively (Figs. $2 \mathrm{C}$ and $2 \mathrm{D}$ ).

Unlike RFS, tumor size, LN classification and molecular type did not affect the risk of CBC. BRCA mutation was the only independent risk factor of developing $C B C$ in the analysis of the overall patients.

\section{Characteristics of $\mathrm{CBC}$ risk in $B R C A$ mutation carriers}

Previous studies reported that patients younger than 40 years old had a higher risk of having CBC [9], so we divided patients into two groups: people at or younger than 40 years-old and people older than 40 years-old. However, the $C B C$ risk was similar between $B R C A$ patients younger and older than 40 years old (Fig. 3A). We found that there was a higher trend of CBC risk in BRCA1 mutation patients (HR=1.814, $95 \% \mathrm{Cl} 0.865-3.805, \mathrm{p}=0.115)$ compared to that of $B R C A 2$ mutation carriers. Similarly, TNBC patients had a higher trend of developing $\mathrm{CBC}$ than $\mathrm{ER}(+) B R C A$ patients $(\mathrm{HR}=1.657,95 \% \mathrm{Cl} 0.778-3.530, \mathrm{p}=$ 0.185 , Fig. $3 B$ ). When genotype and molecular types were integrated, BRCA1 patients with TNBC had the highest risk of $\mathrm{CBC}$ compared to other $B R C A$ mutation patients (Fig. $3 \mathrm{C}$ and $3 \mathrm{D}$ ). The $\mathrm{CBC}$ risk of $B R C A 1$ patients with TNBC was 39.3\% (95\% Cl 19.9\% - 58.7\%) and 63.6\% (95\% Cl 39.0\%-88.1\%) at 10 and 15 years, respectively. In comparison to the control patients, $B R C A 1$ patients with TNBC had a greater than five-fold risk of developing CBC $(H R=5.550,95 \%$ Cl 3.295-9.349, $p<0.001)$.

Figure 3 Cumulative incidence of $\mathrm{CBC}$ in patients among $B R C A$ mutation. The $\mathrm{x}$-axis is followup time (years) and y-axis is cumulative incidence of CBC. (A) Risk of $C B C$ by age onset of the first breast cancer (Less or equal to 40 year-old vs. more than 40 year-old. (B) Risk of CBC by ER(+) vs. TNBC (C) Risk of CBC by between different genotype and molecular type. (D) Risk of CBC by BRCA1 with TNBC vs. others.</fig>

\section{Relationship Between Survival And Cbc}

For the entire cohort, patients with and without $\mathrm{CBC}$ had a similar RFS ( $\mathrm{HR}=0.760,95 \% \mathrm{Cl} 0.540-1.070)$ and OS ( $\mathrm{HR}=0.864,95 \% \mathrm{Cl} 0.597-1.251)$. For the 128 BRCA mutation patients, 22 patients relapsed; 2 patients relapsed before $\mathrm{CBC}$ and 20 patients relapsed after $\mathrm{CBC}$. For all patients with $\mathrm{CBC}$, the 5-year RFS after CBC was $86.9 \%(95 \% \mathrm{Cl} 82.0 \%-91.8 \%)$.

\section{Discussion}

This is, to the best of our knowledge, the first study to investigate the clinical-pathologic characteristics, survival and risk of $\mathrm{CBC}$ in Asian BRCA-mutated breast cancer patients. The characteristics of early-onset and molecular type of breast cancer in our patients are compatible with results in Western ethnic patients $[4,22]$. We observed similar survival between BRCA mutated and non-mutated breast cancer patients. 
However, a significantly elevated risk of $\mathrm{CBC}$ was noted in $B R C A$-mutated breast cancer patients, especially BRCA1 patients with TNBC.

The risk of $\mathrm{CBC}$ was about four- times higher in $B R C A$ mutation carriers than noncarriers. The cumulative 10-year risk of $\mathrm{CBC}$ was about $26.0 \%$. Prior studies estimated the 10 -year risk of $\mathrm{CBC}$ to be about $21 \%-$ $30 \%$. When the observation period lengthened to 20 years, the risk of $\mathrm{CBC}$ could increase up to about $40 \%$ $[9,23]$. Many studies have investigated CBC risk factors. Graeser MK et al. and van den Broek AJ et al. reported that a younger onset age of the first breast cancer was associated with a significantly elevated risk of $\mathrm{CBC}$ in patients with $B R C A$ mutations $[9,24]$. However, we did not find an elevated risk of $\mathrm{CBC}$ in $B R C A$ patients younger than 40 years old in our present cohort, which may be due to ethnic differences or limited followup time. Additionally, the small number of young-onset patients in our cohort may be another reason why our results showed no difference in CBC risk between patients younger and older than 40 years old. In our cohort, only $39 \%$ of patients were younger than 40 years old when they had their first breast cancer; the median onset age of the first breast cancer in BRCA1 and BRCA2 patients were 42.2 and 45.6 years old, respectively. We are continuing enrolling early onset of Taiwanese breast cancer patients to better understand the epidemiology as well as risk factors of $\mathrm{CBC}$ in Asian breast cancer patients.

However, in concordance with previous studies [9], our data revealed that $B R C A 1$ patients had a higher trend of developing $\mathrm{CBC}$ than $B R C A 2$ patients. When classified by genotype and molecular types, $B R C A 1$ patients with TNBC had the highest risk of getting $\mathrm{CBC}$; the $\mathrm{CBC}$ risk of these patients was up to $63.6 \%$ $(95 \% \mathrm{Cl} 39.0 \%-88.1 \%)$ at 15 years, about a five-fold increase in comparison to control patients. BRCA1 patients with $\mathrm{ER}(+)$ breast cancer had similar risk of developing $\mathrm{CBC}$ compared to whole BRCA2 patients (Fig. 3C).

The ASCO consensus and NCCN guidelines suggest that risk-reducing mastectomy and contralateral prophylactic mastectomy (CPM) should be considered for $B R C A$ mutation carriers [19, 25]. CPM can reduce the risk of $\mathrm{CBC}$. Some studies even reported that $\mathrm{CPM}$ can reduce mortality for $B R C A$ mutation patients in the long-term follow-up [26-28]. However, the CPM rate was relative lower in Asian countries[29]. In our study, only two BRCA mutation patients received CPM; this is one of the reasons of the high $\mathrm{CBC}$ risk in our cohort. Therefore, $\mathrm{CPM}$ will be considered for our patients to reduce the risk of $\mathrm{CBC}$, especially for BRCA1 patients with TNBC.

The impact of germline BRCA mutations on the survival of breast cancer patients is controversial. A prospective study revealed that patients with young-onset breast cancer who carry a $B R C A$ mutation have similar survival as non-carriers[11]. Another large cohort study showed that $B R C A 1$ mutation carriers, independent of clinical-pathological and therapy characteristics, had a worse overall survival (OS) than noncarriers[13]. Subsequent ovarian cancer in BRCA1 breast cancer survivors may contribute to the lower $\mathrm{OS}$. The breast cancer mortality rate was not significantly superior in $B R C A$ mutation patients[13]. Our study demonstrated that BRCA breast cancer patients had a similar RFS in comparison to that of control patients. However, the risk of non-breast second malignancies, especially ovarian cancer, was 
significantly higher than that of control patients. Besides from ovarian cancer, we also saw two cases of endometrial cancer. One patient's endometrial cancer received multi-gene sequencing and the result showed loss-of-heterozygosity of the BRCA1 gene, indicating that BRCA1 mutation was involved in tumorigenesis. Furthermore, several recent studies found an increased risk of uterine cancer in $B R C A$ mutation carriers $[30,31]$. These facts suggested that the prevention of second malignancies is an important issue in BRCA breast cancer survivors.

Asians comprise of $60 \%$ of the world's population and this number is still increasing. A prior review study revealed that Asian $B R C A$ patients had a similar epidemiology and mutation spectrum compared to Western countries [15]; that fact implies that most BRCA mutation carriers are Asian. In this study, we provided evidence that our $B R C A$ patients had an increased risk of $\mathrm{CBC}$ and second malignancies. The phenomena resemble the cancer risks of other ethnic groups, suggesting that Asian BRCA carriers are a high-risk population susceptible to breast cancer, $\mathrm{CBC}$ and second malignancies. In particular, we found that BRCA1 patients with TNBC as their first cancer had a greater than five-fold risk of developing CBC. Thus, genetic counseling and management of Asian BRCA mutation carriers needs to improve in the future.

\section{Declarations}

\section{Acknowledgment}

We thank the patients, physicians, clinical genetic counselor and nurses who participated in this study to establish the Taiwan BRCA cohort.

\section{Funding}

This work was supported, in part, by research grants from the National Taiwan University Hospital (NTUH. 107-004068) and the Ministry of Science and Technology (MOST 104-2314-B-002-106-MY3 and MOST 109WFA0111726).

\section{Data availability}

The datasets used during the current study are available from the corresponding author on reasonable request.

\section{Author information}

\section{Affiliations}

National Taiwan University Hospital, Taipei, Taiwan

Po-Han Lin, King-Jen Chang, Ai-Chu Huang, Kuo-Chih Cheng, Wen-Hung Kuo, Ching-Hung Lin, Huo-Mu Chen, Ming-Yang Wang, Chiao Lo, Yen-Shen Lu and Chiun-Sheng Huang 
Chang Gung Memorial Hospital

Shin-Cheh Chen, Wen-Lin Kuo

Taipei Veterans General Hospital, Taipei, Taiwan

Ling-Ming Tseng, Chun-Yu Liu

Princeton University, Princeton, New Jersey, USA

Karen Yang

Columbia University Mailman School of Public Health, New York, USA

Hui-Chen Wu

Cheng Ching Hospital, Taichung, Taiwan

Dah-Cherng Yeh

China Medical University Hospital, Taichung, Taiwan

Liang-Chih Liu

Shuang-Ho Hospital, Taipei Medical University, Taiwan

Tsu-Yi Chao

MacKay Memorial Hospital, Taipei, Taiwan

Yuan-Ching Chang

National Cheng Kung University, Tainan, Taiwan.

Peng-Chan Lin

Corresponding authors

Correspondence to Chiun-Sheng Huang.

\section{Ethics declarations}

Conflict of interest

All the authors declare that they have no conflict of interest. 
BRCA mutation patients were collected from two studies, which were approved by the Medical Ethics Committee of the National Taiwan University Hospital (201308077RINA and 201706090RINA). Ninety patients received gene diagnosis at National Taiwan University Hospital. Although other patients were diagnosed at other hospitals, most of the BRCA mutation carriers were refereed to genetic clinic of the National Taiwan University Hospital for genetic counseling so we could collect their data.

\section{Informed consent}

In this study, all procedures were performed in accordance with the Declaration of Helsinki.

\section{References}

1. Siegel RL, Miller KD, Jemal A (2020) Cancer statistics, 2020. CA Cancer J Clin 70:7-30

2. Kuo WH, Lin PH, Huang AC et al (2012) Multimodel assessment of BRCA1 mutations in Taiwanese (ethnic Chinese) women with early-onset, bilateral or familial breast cancer. J Hum Genet 57:130138

3. Lin PH, Kuo WH, Huang AC et al (2016) Multiple gene sequencing for risk assessment in patients with early-onset or familial breast cancer. Oncotarget 7:8310-8320

4. Kuchenbaecker KB, Hopper JL, Barnes DR et al (2017) Risks of Breast, Ovarian, and Contralateral Breast Cancer for BRCA1 and BRCA2 Mutation Carriers. JAMA 317:2402-2416

5. Mavaddat N, Peock S, Frost D et al (2013) Cancer risks for BRCA1 and BRCA2 mutation carriers: results from prospective analysis of EMBRACE. J Natl Cancer Inst 105:812-822

6. Park B, Dowty JG, Ahn C et al (2015) Breast cancer risk for Korean women with germline mutations in BRCA1 and BRCA2. Breast Cancer Res Treat 152:659-665

7. Zhang L, Shin VY, Chai $X$ et al (2018) Breast and ovarian cancer penetrance of BRCA1/2 mutations among Hong Kong women. Oncotarget 9:25025-25033

8. Pierce LJ, Levin AM, Rebbeck TR et al (2006) Ten-year multi-institutional results of breast-conserving surgery and radiotherapy in BRCA1/2-associated stage I/II breast cancer. J Clin Oncol 24:2437-2443

9. van den Broek AJ, van 't Veer LJ, Hooning MJ et al (2016) Impact of Age at Primary Breast Cancer on Contralateral Breast Cancer Risk in BRCA1/2 Mutation Carriers. J Clin Oncol 34:409-418

10. Valachis A, Nearchou AD, Lind P (2014) Surgical management of breast cancer in BRCA-mutation carriers: a systematic review and meta-analysis. Breast Cancer Res Treat 144:443-455

11. Copson ER, Maishman TC, Tapper WJ et al (2018) Germline BRCA mutation and outcome in youngonset breast cancer (POSH): a prospective cohort study. Lancet Oncol 19:169-180

12. Zhong Q, Peng HL, Zhao X et al (2015) Effects of BRCA1- and BRCA2-related mutations on ovarian and breast cancer survival: a meta-analysis. Clin Cancer Res 21:211-220

13. Schmidt MK, van den Broek AJ, Tollenaar RA et al. Breast Cancer Survival of BRCA1/BRCA2 Mutation Carriers in a Hospital-Based Cohort of Young Women. J Natl Cancer Inst 2017; 109 
14. Kang E, Park SK, Lee JW et al (2016) KOHBRA BRCA risk calculator (KOHCal): a model for predicting BRCA1 and BRCA2 mutations in Korean breast cancer patients. J Hum Genet 61:365-371

15. Kwong A, Shin VY, Ho JC et al (2016) Comprehensive spectrum of BRCA1 and BRCA2 deleterious mutations in breast cancer in Asian countries. J Med Genet 53:15-23

16. Arai M, Yokoyama S, Watanabe $C$ et al (2018) Genetic and clinical characteristics in Japanese hereditary breast and ovarian cancer: first report after establishment of HBOC registration system in Japan. J Hum Genet 63:447-457

17. Dong H, Chandratre K, Qin Y et al. Prevalence of BRCA1/BRCA2 pathogenic variation in Chinese Han population. J Med Genet 2020

18. Wen WX, Allen J, Lai KN et al (2018) Inherited mutations in BRCA1 and BRCA2 in an unselected multiethnic cohort of Asian patients with breast cancer and healthy controls from Malaysia. J Med Genet 55:97-103

19. Daly MB, Pilarski R, Yurgelun MB et al (2020) NCCN Guidelines Insights: Genetic/Familial High-Risk Assessment: Breast, Ovarian, and Pancreatic, Version 1.2020. J Natl Compr Canc Netw 18:380-391

20. Ehinger A, Malmstrom P, Bendahl PO et al (2017) Histological grade provides significant prognostic information in addition to breast cancer subtypes defined according to St Gallen 2013. Acta Oncol $56: 68-74$

21. Goldhirsch A, Winer EP, Coates AS et al (2013) Personalizing the treatment of women with early breast cancer: highlights of the St Gallen International Expert Consensus on the Primary Therapy of Early Breast Cancer 2013. Ann Oncol 24:2206-2223

22. Mavaddat N, Barrowdale D, Andrulis IL et al (2012) Pathology of breast and ovarian cancers among BRCA1 and BRCA2 mutation carriers: results from the Consortium of Investigators of Modifiers of BRCA1/2 (CIMBA). Cancer Epidemiol Biomarkers Prev 21:134-147

23. Pierce LJ, Phillips KA, Griffith KA et al (2010) Local therapy in BRCA1 and BRCA2 mutation carriers with operable breast cancer: comparison of breast conservation and mastectomy. Breast Cancer Res Treat 121:389-398

24. Graeser MK, Engel C, Rhiem K et al (2009) Contralateral breast cancer risk in BRCA1 and BRCA2 mutation carriers. J Clin Oncol 27:5887-5892

25. Tung NM, Boughey JC, Pierce LJ et al (2020) Management of Hereditary Breast Cancer: American Society of Clinical Oncology, American Society for Radiation Oncology, and Society of Surgical Oncology Guideline. J Clin Oncol 38:2080-2106

26. Heemskerk-Gerritsen BA, Rookus MA, Aalfs CM et al (2015) Improved overall survival after contralateral risk-reducing mastectomy in BRCA1/2 mutation carriers with a history of unilateral breast cancer: a prospective analysis. Int J Cancer 136:668-677

27. Metcalfe K, Gershman S, Ghadirian P et al (2014) Contralateral mastectomy and survival after breast cancer in carriers of BRCA1 and BRCA2 mutations: retrospective analysis. BMJ 348:g226

28. van Sprundel TC, Schmidt MK, Rookus MA et al (2005) Risk reduction of contralateral breast cancer and survival after contralateral prophylactic mastectomy in BRCA1 or BRCA2 mutation carriers. $\mathrm{Br} J$ 
Cancer 93:287-292

29. Jeon HJ, Park HS, Park JS et al (2019) Trends in contralateral prophylactic mastectomy rate according to clinicopathologic and socioeconomic status. Ann Surg Treat Res 97:113-118

30. Laitman Y, Michaelson-Cohen R, Levi E et al (2019) Uterine cancer in Jewish Israeli BRCA1/2 mutation carriers. Cancer 125:698-703

31. Shu CA, Pike MC, Jotwani AR et al (2016) Uterine Cancer After Risk-Reducing Salpingooophorectomy Without Hysterectomy in Women With BRCA Mutations. JAMA Oncol 2:1434-1440

\section{Figures}


A

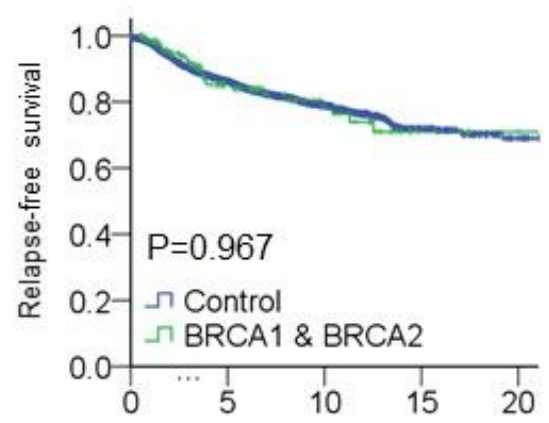

C
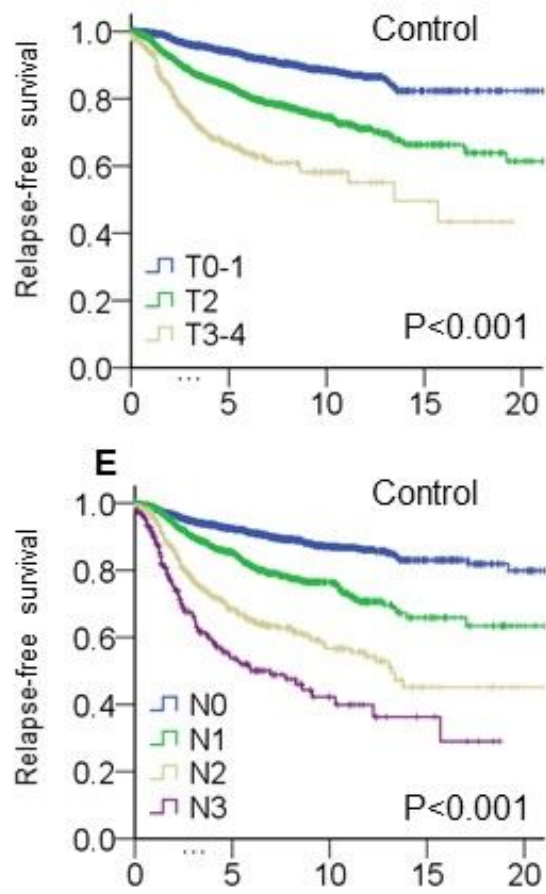

G

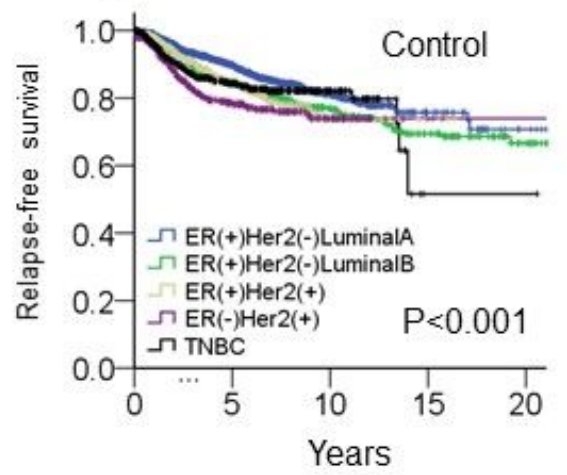

B

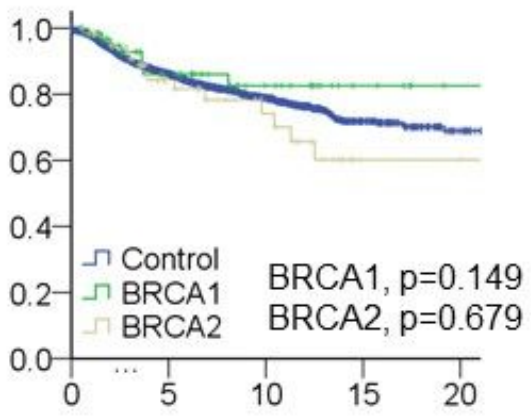

D
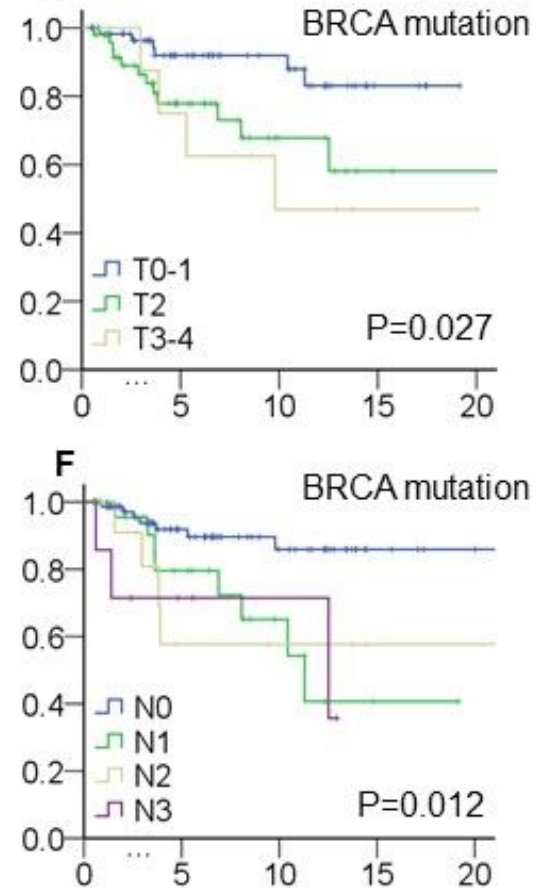

H

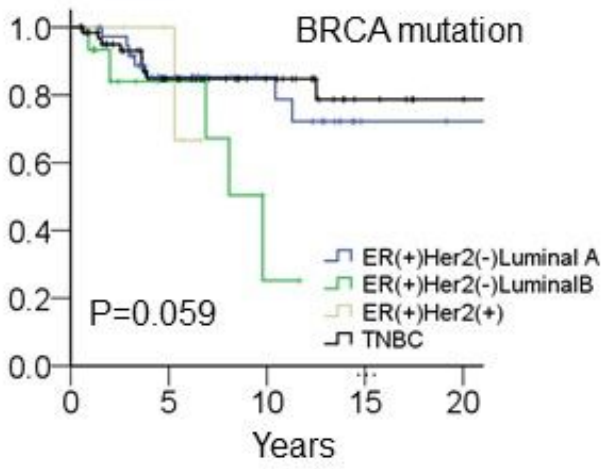

\section{Figure 1}

Relapse-free survival analysis by different characteristics. The x-axis is followup time (years) and y-axis is cumulative relapse-free survival. (A) Kaplan-Meier analysis of relapse-free survival by BRCA mutation (Control patients versus (vs.) patients with BRCA1 \&2 mutation). (B) Kaplan-Meier analysis of relapse-free survival by different BRCA mutation groups (Control patients vs. patients with BRCA1 and Control patients vs BRCA2 mutation). (C) Kaplan-Meier analysis of relapse-free survival by $T$ classification 
among control patients. (D) Kaplan-Meier analysis of relapse-free survival by T classification among patients with BRCA1 \&2 mutation. (E) Kaplan-Meier analysis of relapse-free survival by $\mathrm{N}$ classification among control patients. (F) Kaplan-Meier analysis of relapse-free survival by $\mathrm{N}$ classification among patients with BRCA1 \&2 mutation. (G) Kaplan-Meier analysis of relapse-free survival by molecular type among control patients. $(\mathrm{H})$ Kaplan-Meier analysis of relapse-free survival by molecular type among patients with BRCA1 \&2 mutation.

A

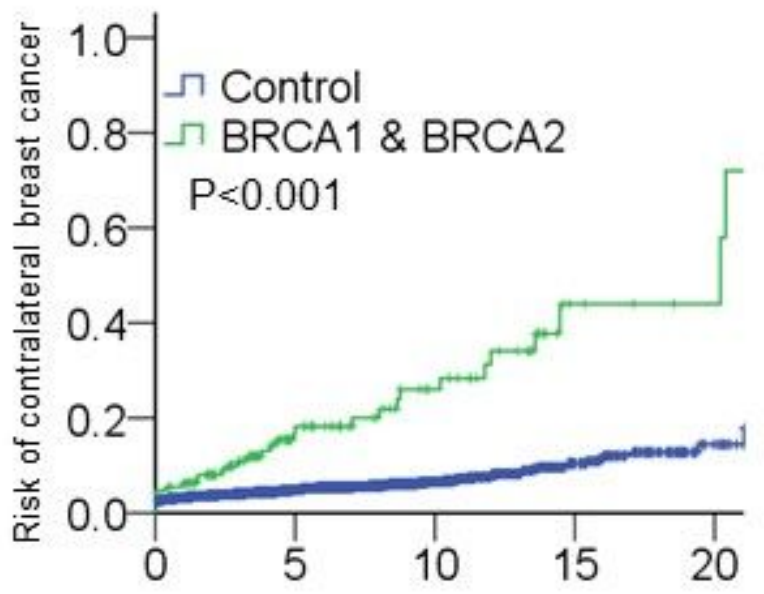

C

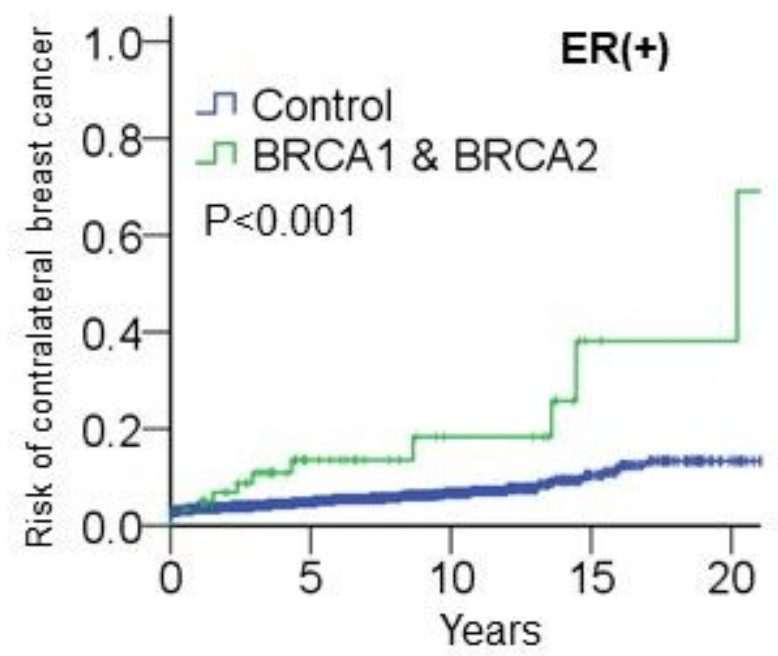

B

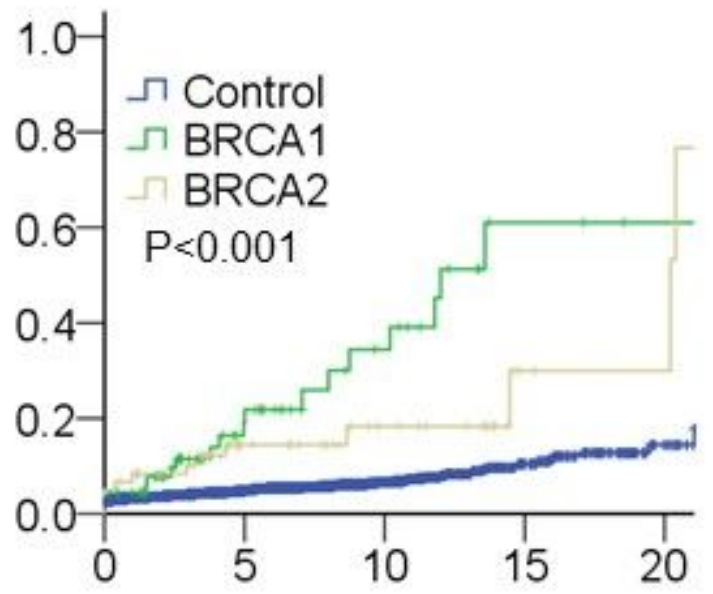

D

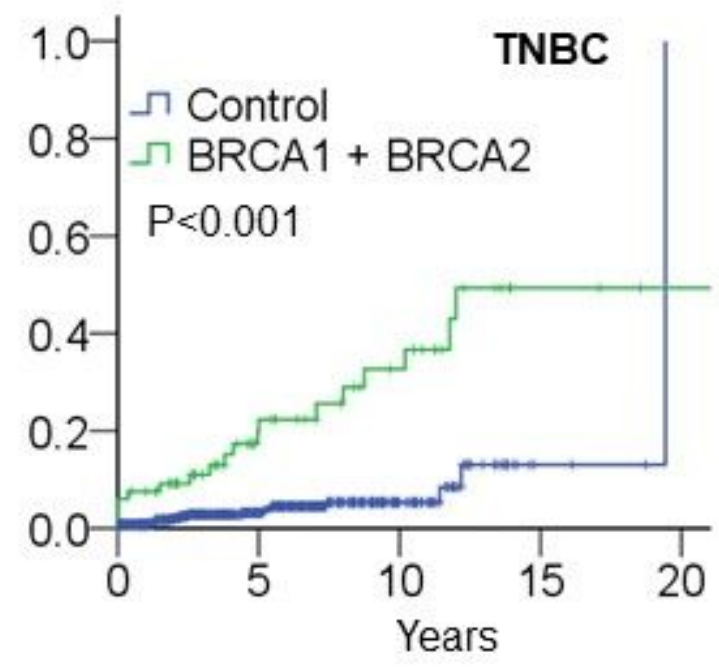

\section{Figure 2}

Cumulative incidence of contralateral breast cancer (CBC). The $x$-axis is followup time (years) and y-axis is cumulative incidence of $C B C$. (A) Risk of $C B C$ by BRCA mutation (Control patients versus (vs.) patients with BRCA1 \&2 mutation). (B) Risk of CBC by different BRCA mutation groups (Control patients vs. patients with BRCA1 and Control patients vs BRCA2 mutation). (C) Risk of $C B C$ among ER (+) by BRCA mutation (Control patients versus (vs.) patients with BRCA1 \&2 mutation). (D) Risk of CBC among TBNC patients by BRCA mutation (Control patients versus (vs.) patients with BRCA1 \&2 mutation). 


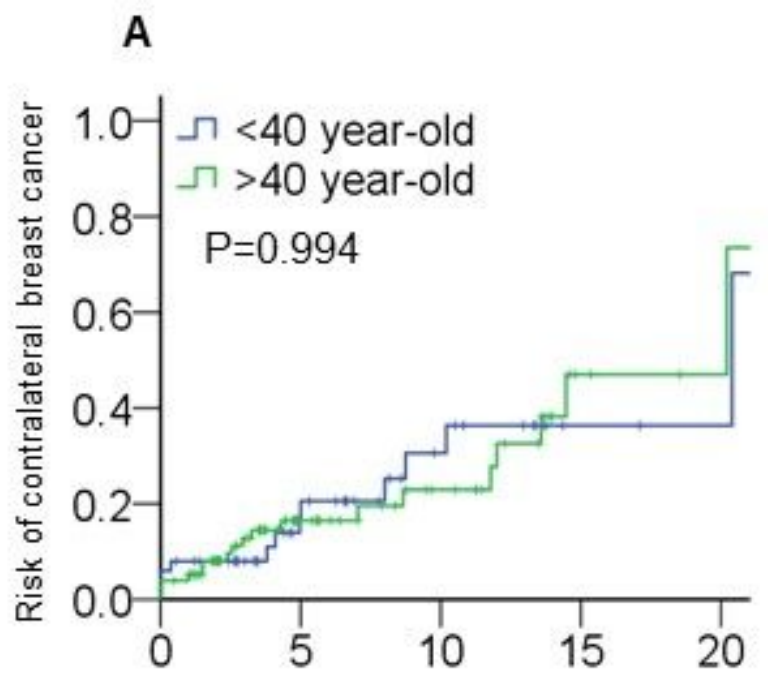

B

C
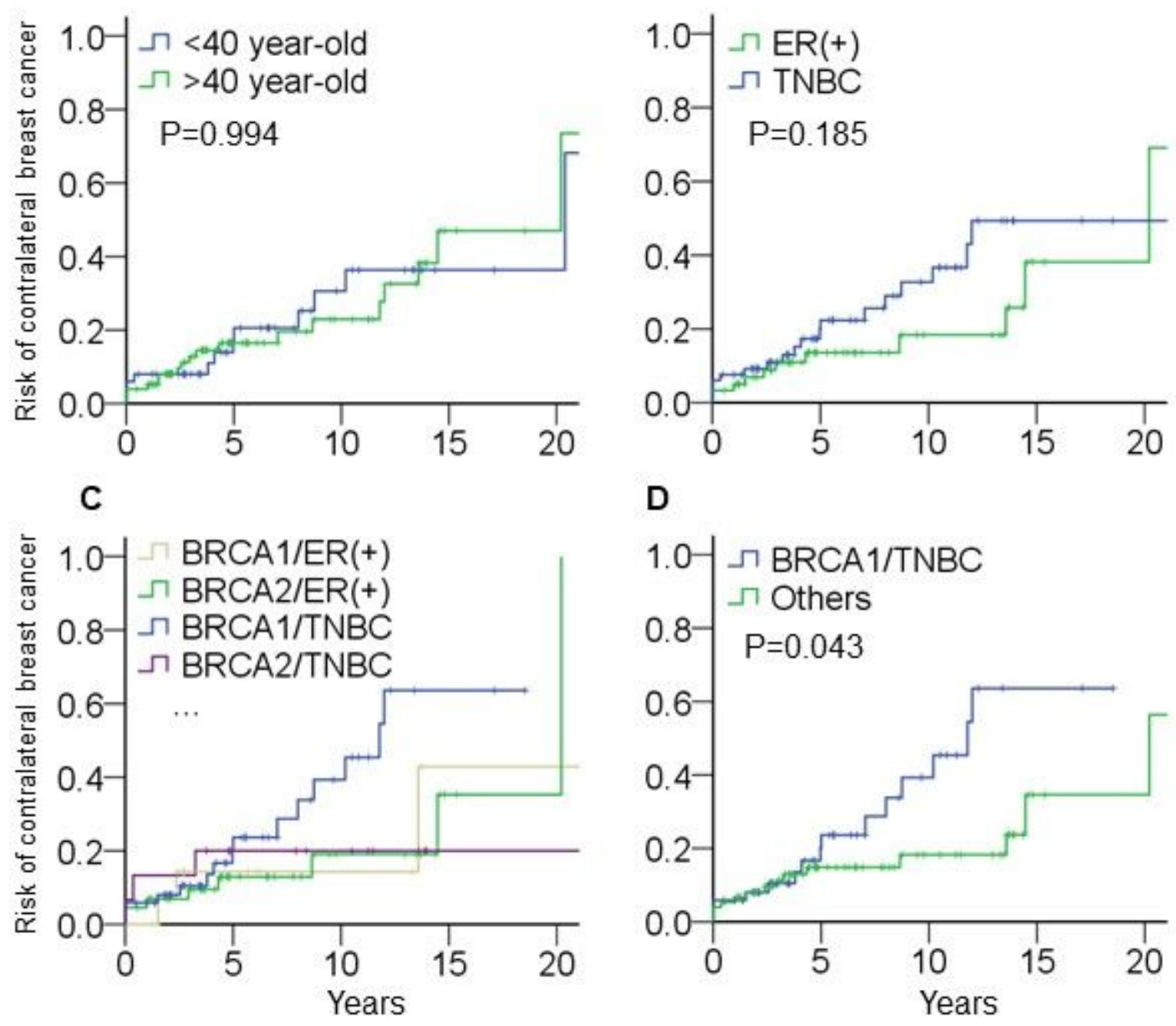

D

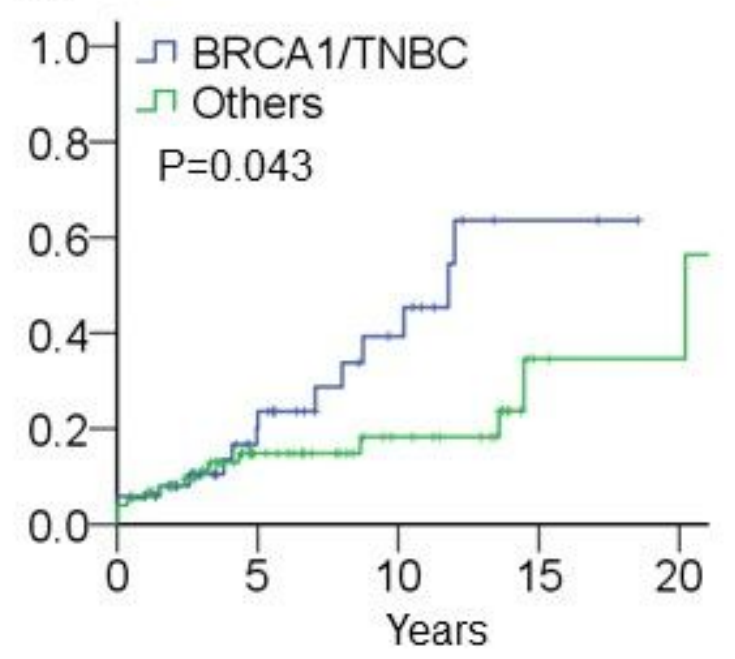

\section{Figure 3}

Cumulative incidence of $C B C$ in patients among BRCA mutation. The $x$-axis is followup time (years) and $y$-axis is cumulative incidence of CBC. (A) Risk of CBC by age onset of the first breast cancer (Less or equal to 40 year-old vs. more than 40 year-old. (B) Risk of $C B C$ by ER(+) vs. TNBC (C) Risk of $C B C$ by between different genotype and molecular type. (D) Risk of CBC by BRCA1 with TNBC vs. others. 